\title{
Familia y Tercera Edad: variables predictoras del abandono del anciano
}

\author{
Miguel Ángel Vidal ${ }^{1}$ \\ Miguel Clemente ${ }^{2}$
}

\begin{abstract}
Resumo
Neste artigo abordaremos a problemática dos idosos em uma sociedade na qual é cada vez maior o número de pessoas com idade superior a 65 anos. Uma das consequiências do aumento da média de idade e de uma maior qualidade de vida dos idosos é, precisamente, a não existência de pessoas que os assumam. Estudaremos, nestas linhas, o risco de abandono e quais são as variáveis mais importantes na predição deste risco. Para tanto trabalharemos com dois grupos de idosos (os que vivem em casa com seus familiares e os que vivem em residências para idosos), o que totaliza 121 idosos e, mediante a técnica estatística da regressão logística obteremos as variáveis com maior peso para discriminar entre os dois grupos e predizer o risco de abandono.
\end{abstract}

Palavras-Chave: idoso; abandono; família.

\section{Abstract}

This paper approaches the problem of the elderly in a society of a growing number of people who are more than 65 years old. One of the consequences of a higher average age and a better quality of life of the elderly is, precisely, the lack of people who assume them. Following these lines, this study concerns the risk of abandonment and the most important variables in the prediction of this risk. Two elderly groups (those that live at home with their families and those that live in rest homes) of a total of 121 elders is the studied population.

\footnotetext{
1 Doctor en Psicología. Profesor de Psicología Social. Universidad Pontificia de Salamanca. España. Email:mavidal@eucmos.sim.ucm.es

2 Doctor en Psicología. Profesor Titular de Psicología Social. Universidad de A Coruña. España. E-mail: clemen@udc.es
}

Estud. interdiscip. envelhec., Porto Alegre, v.2, p.49-65, 1999. 
By means of the statistics technique of logistic regression, the variables with greater influence to discriminate between the two groups and predict the risk of abandonment were obtained.

Key-Words: elderly; abandonment; family.

\section{Introducción}

Si bien el abandono de ancianos como tal, es bastante difícil que se produzca en nuestros días, y los pocos casos existentes no se pueden considerar como problemas sociales, sí es cierto la existencia de un abandono subjetivo. Entendemos por abandono subjetivo, la percepción que el anciano tiene de su situación social, que si bien físicamente está rodeado de personas (hijos, nietos, amigos, vecinos, asistentes sociales, entre otros muchos), su percepción de la realidad es de soledad y abandono. A lo largo de estas páginas realizaremos una revisión sobre el tema tanto del abandono real como del subjetivo, tratando de encontrar cuáles podrían ser la variables relevantes implicadas en ambos fenómenos.

Pero situémonos en la realidad a estudiar: el anciano. A las puertas del siglo XXI, las sociedades desarrolladas, entre las que nos encontramos, están sufriendo un fenómeno de envejecimiento poblacional. Esto, unido a una mayor calidad de vida y a un incremento notable en nuestra esperanza de vida, hace que la situación social de finales de los 90 sea muy distinta a la de tan sólo unas décadas atrás. Hoy en día no es raro encontrarse con ancianos que superan los 80 años, que son cuidados por sus hijos, que rondan los 60 años de edad. Y es aquí donde se encuentran la mayoría de los estudios sobre el tema.

Las familias que cuidan de sus mayores, se han convertido en el objeto de estudio actual, siendo el principal objetivo de los investigadores evaluar a las familias en el desempeño de su nueva tarea (Dwyer; Folts; Rosenberg, 1994; Phillips; Rempusheski; Morrison, 1989), o tratando de predecir el riesgo de abandono total o parcial de los mayores, entendiendo por parcial la reclusión del anciano en residencias, contra su voluntad aunque reciba visitas de sus familiares (véase, por ejemplo las investigaciones de Clemente; Vidal; Reguera, 1997). Este tipo de trabajos tratan de dilucidar cuáles son los factores que más 
influyen en las familias a la hora de plantearse el abandono como la mejor alternativa, así como las variables o situación de la persona mayor que facilita esta decisión (por ejemplo Thompson; Futterman; Gallagher-Thompson; Rose; Lovett, 1993; Wilber; Specht, 1994). Las variables que se contemplan en estos estudios se refieren fundamentalmente a la situación sociodemográfica de la familia, el contexto ambiental cercano, las condiciones de habitabilidad de la vivienda, los factores de comunicación e integración familiar, la existencia de otras relaciones sociales, o la forma en que los ancianos socializaron a sus hijos. Pero también se estudia al grado de asistencia de los familiares al anciano, pero recogiendo la información de los propios familiares, no del anciano (Dietz, 1995).

\section{Conceptualización y dimensión de la ancianidad}

Los diferentes términos utilizados desde un punto de vista social para nombrar la etapa de la vida que nos ocupa (anciano, viejo, tercera edad entre nosotros), no son expresiones opuestas, sino en realidad complementarias y explicables en función del punto de vista que se adopte a la hora de explicar la misma. Y la verdad es que a veces se trata, desde un punto de vista terminológico, sólo de pequeños matices; pero que implican una visión de la realidad muy diferente.

Desde un punto de vista científico, también son varias las razones según las cuales los estudiosos justifican el tratamiento del tema; paran algunos, la justificación se centra en el interés del estudio del paso del tiempo, utilizando por lo tanto una visión cronológica del problema. Pero no es ésta, por ejemplo, la visión de la Organización Mundial de la Salud, que define al viejo como aquel que no puede vivir de forma autónoma, ya que sus capacidades físicas o mentales han disminuido de forma notable; en una palabra, el factor explicativo se sitúa en la función. No han faltado quienes han utilizado una visión mercantilista del término, y han asociado el término viejo a la persona improductiva. De manera general, es preciso señalar que desde un punto de vista científico los mayores nunca han salido muy bien parados.

Quizá una pequeña aportación a una visión más positiva del tratamiento científico del problema la encontramos en algunas perspectivas de la Psicología 
del Desarrollo, llegando a afirmar algunos autores que comenzamos a envejecer cuando nacemos; perspectiva que sitúa la vejez como una denominación más del continuo de la vida del individuo, siendo esta perspectiva la visión de la orientación del "desarrollo de la vida".

La valoración del anciano ha variado a lo largo de la Historia. Cuestión lógica a grandes rasgos, ya que, por ejemplo, en nuestra sociedad en momentos anteriores al actual, la consideración de la vejez ha sido muy positiva, y lo sigue siendo en muchas culturas. Pero si hablamos de valoración, es preciso reconocer que el anciano se identifica a sí mismo y se conceptualiza de acuerdo con los demás. Su imagen es social como todas; como la de los niños o la de cualquiera; al fin y al cabo, todos somos animales sociales.

Por su parte, afirmaciones como las de Alfred Soury " ( . . . ) el envejecimiento es el problema más grave del siglo" (López Jiménez, 1993), nos hacen ver y comprender cómo nos encontramos ante todo un problema social. Razón de más si, como es el caso de este capítulo, nos centramos en un tema tan específico como es el de las redes de apoyo social y el sentimiento de abandono.

Pero existen una serie de datos estadísticos que además, verifican esta idea. Así, si examinamos la evolución del cociente entre ancianos frente a la población total, podemos comprobar que sólo en la Comunidad de Madrid, se ha ido evolucionando desde un pequeño $1 \%$ en 1900 , hasta un $12 \%$ previsto para el año 2001 (COMUNIDAD DE MADRID, 1989). En regiones y países centro y norte europeos, el problema resulta aún mucho más grave.

Lo cierto es que cualquier pirámide de población que estudiemos hoy en día muestra el progresivo envejecimiento de nuestra población, y la erosión causada en edades tempranas; cada vez tenemos menos niños, cada vez tenemos más ancianos. Hoy en día las sociedades pueden considerarse jóvenes cuando menos del 5\% de su población total es anciana; de tipo medio cuando dicha tasa se sitúa entre un 5 y un 10\%; y envejecida como la nuestra, cuando se supera el $10 \%$ de población anciana, siendo además este fenómeno el más común y apareciendo las denominadas pirámides invertidas de población: menor número de nacimientos que de ancianos.

Sin duda en este fenómeno ha tenido mucho que ver el incremento de la 
denominada esperanza de vida, a la que hacíamos referencia anteriormente. Es decir, los años que presumiblemente vivirá una persona en una región determinada. En España, esa esperanza de vida en el año 1994 era de 77.6 años (EL PAIS, 1998). Por sexos, nos encontramos que esta evolución es mayor en las mujeres que en los hombres, pasando desde los 35.7 años en 1900, hasta los 79.6 años en 1989; y en el caso de los varones, desde los 33.9 años a principios de siglo a los 73.1; es decir, en general ahora se vive el doble de lo que se vivía hace 100 años.

Este espectacular aumento de la esperanza de vida ha supuesto el nacimiento de nuevas nomenclaturas entre los estudiosos de la demografía. Y así se crea la etiqueta de la cuarta edad, para aquellos que superan los 80 años. Además, haciendo proyecciones demográficas, se estima que en España (de seguir las cosas evolucionando como hasta ahora) tendremos en el año 2010 un total de 8.350 .000 personas con una edad igual o superior a los 65 años de edad. Este resultado indica que para tal fecha, uno de cada cinco españoles (el $20 \%$ de la población) será anciana.

\section{Los ancianos en nuestra sociedad}

$\mathrm{El}$ anciano es un ser que ha sido despedido del mundo productivo. Esto supone una pérdida de rol, que en ocasiones se una a una pérdida de seres queridos, a menudo incluyendo a su cónyuge. Normalmente, el fin de la etapa laboral le va a suponer la pérdida, muchas veces traumática, de los contactos personales con los compañeros, una pérdida de su estatus, de prestigio, y en muchos casos, una bajada importante de la autoestima, creyéndose que no vale nada. Otros elementos corroboran este proceso de índole negativa, agudizándolo; así, el anciano ve mermados sus ingresos económicos, lo que supone que debe adaptarse a otro nivel de gastos. Por otra parte, el anciano se encuentra de repente con una gran cantidad de tiempo libre, debiendo aprender a reorganizarlo.

Algunos autores han resaltado estas cuestiones bajo la rúbrica de la desvinculación, caracterizada fundamentalmente por dos notas características: la disminución de los contactos sociales, y también de los compromisos emocionales. La desvinculación presenta tanto características positivas como 
negativas; entre los aspectos positivos se podrían destacar los siguientes:

- recuperación del tiempo libre;

— posibilidades de dedicarse a tareas más creativas;

— recuperación de la libertad.

Pero también presenta una serie de consecuencias negativas, como las siguientes que se destacan a continuación:

— disminución de los ingresos y de las posibilidades económicas;

— disminución de las capacidades físicas;

- disminución de las capacidades mentales y psicológicas.

Frente a esta situación, algunos ancianos optarán por aislarse; otros, por "vivir" más la familia junto con sus hijos y nietos. Y otros, por acudir a los centros de servicios sociales, clubes sociales creados para ancianos, etc.

Pero veamos cómo el anciano es visto por su propio entorno y cuál es su función. Los datos que aquí mostraremos se refieren fundamentalmente a un estudio realizado por el AYUNTAMIENTO DE MADRID (1993), sobre el papel que tiene el anciano en la actualidad dentro de las familias. Se trata de una encuesta llevada a cabo entre escolares de Madrid capital, que estaban estudiando el ciclo superior de Educación General Básica y de Bachillerato Unificado Polivalente, con un rango de edad desde los 11 a los 16 años.

Entre estos niños, el 95\% de los encuestados informó tener vivo algún abuelo y relacionarse alguna vez con ellos o con abuelos de otros compañeros; es decir, la mayoría de las personas que iban a responder poseían un contacto con personas mayores, por lo que tenían conocimiento de causa sobre lo que iban a contestar.

Establecido este punto, una de las preguntas que se les realizaba se refería a las actividades que realizan con ellos; la mayoría de ellos hablaban del cuidado personal, también de ir de compras, y un $44 \%$ decía jugar con ellos.

Por otra parte, si tratamos de establecer la imagen que los jóvenes tienen de sus ancianos, la cualidad que más destacan es la de que son agradables, seguida de la de cariñosos, cuidadores, proporcionadores de regalos, o de enseñanzas. Todo esto nos hace pensar que para los jóvenes son importantes los ancianos, quizá fruto de una existencia acelerada en las familias, donde la 
permanencia del padre y de la madre ha disminuido, y por lo tanto los abuelos asumen una labor de mayor presencia familiar.

Resulta curioso también observar cuáles creen los jóvenes que son los principales problemas de los ancianos; frente a la posible idea de que la enfermedad podría ser el factor más preocupante, nos encontramos que no es así, destacando sobre todo la soledad como factor clave, seguida por el abandono y también por la dependencia dentro de la familia. Por lo tanto, los problemas de interrelación se convierten, a juicio de los jóvenes, en los principales para los ancianos.

Abordaremos a continuación esta problemática: el apoyo social. Posteriormente, una vez tratado el tema de las redes sociales y el apoyo social, trataremos la problemática más específica del abandono.

\section{El apoyo social}

\subsection{Enfoques teóricos del apoyo social}

El concepto de apoyo social ha sido uno de los términos más relacionados con el estrés y con la salud, tanto física como psíquica (Brown; Andrews, 1986). Aunque son muchas las concepciones sobre el apoyo social, dos enfoques teóricos son los que nos parecen más adecuados: el enfoque estructural y el enfoque funcional.

El enfoque estructural ha sido defendido a través del concepto de redes sociales (Wellman, 1985). De este modo, el apoyo social viene determinado por las características de la red social. Características tales como el tamaño, la densidad, la composición y la interconexión de sus miembros. El enfoque de las redes sociales asume que los beneficios de la red social de una persona son directamente proporcionales a su tamaño y que tener una relación es equivalente a obtener apoyo de esa relación. Sin embargo, las redes sociales (como ocurre en algunos casos de los ancianos) pueden ser estresantes en sí mismas y ser conflictivas en lugar de prestar ayuda al individuo.

Por su parte, en el enfoque funcional no se enfatiza la estructura del contexto social donde la persona está inmersa sino el tipo de ayuda que los miembros de la red social prestan al individuo, como un factor importante en el ajuste del sujeto. 
Estas dos concepciones dan lugar a dos visiones diferentes dentro del apoyo social: el apoyo real y el apoyo percibido. El apoyo social real da prioridad a la ayuda que, de hecho, el sujeto recibe. Por su parte la idea del apoyo social percibido da prioridad a la percepción que el individuo tiene de su entorno social como potencialmente servicial para él, así como a la satisfacción que el sujeto tiene respecto a esta disponibilidad de apoyo.

\subsection{El apoyo social y el sentimiento de abandono}

La magnitud del esfuerzo de los cuidadores y su coste, son considerables. De este modo, es común y normal que los familiares que tienen ancianos a su cargo, paguen un elevado precio por dichos cuidados: como media, estas familias pasan cuatro horas diarias durante los siete días de la semana dedicados exclusivamente al cuidado de los mayores (Stone; Cafferata; Sangl, 1987). Y no todos los costes son tan fáciles de cuantificar como el del tiempo.

Pero estas cargas pueden ser amortiguadas. Existe una amplia evidencia empírica que demuestra cómo el alto precio de los cuidados se ve reducido gracias al apoyo social (George; Gwyther, 1986; Matthews; Werkner; Delaney, 1989; Pearlin; Mullan; Semple; Skaff, 1990). Aunque sí podemos hablar de un gran consenso en reconocer el apoyo social como un factor muy importante en la reducción de la carga de los cuidadores, no existe acuerdo sobre cómo aminora la experiencia negativa de los cuidadores (ver por ejemplo la revisión de Dunkel-Schetter; Bennett, 1990). También es necesario entender si una clase de apoyo social mediatiza la carga y los costes interpersonales mejor que otros tipos de apoyo. De este modo, la pregunta fundamental no sería cuáles son los tipos de apoyo social que afectan a la experiencia de las familias, sino cómo lo hacen. Además, se suele confundir el concepto de apoyo social con el de redes sociales, por lo que es difícil conocer con exactitud sus efectos sobre las personas que cuidan de los ancianos. De este modo, según House; Umberson; Landis (1988), las redes describen las relaciones sociales de los cuidadores, atendiendo a conceptos tales como densidad, composición y multiplicidad. Pero no todas las relaciones dentro de una red aportan apoyo, ni tampoco una misma clase de soporte social.

Podríamos afirmar, al igual que Thompson et al. (1993); Wellman; 
Wortley (1990), que el apoyo social constituye las siguientes formas primarias: apoyo informal, asistencia, apoyo emocional, estima e integración social. Estos cinco aspectos básicos reducen el sentimiento de carga que los cuidadores pueden tener, como consecuencia de las atenciones que le prestan a los ancianos que están a su cargo.

El apoyo informal hace referencia a los consejos y apoyos que el cuidador recibe por parte de los otros, para entender y manejar las situaciones estresantes. La asistencia sería un conducta instrumental, la cual directamente apoya al cuidador en las tareas que tiene que desempeñar. El apoyo emocional se entiende como la conducta de los otros que proporciona sobre los cuidadores sentimientos de confort, tranquilidad y seguridad. La estima sería un refuerzo positivo para el cuidador, fruto de su buen hacer y por los servicios prestados al anciano. Por último, por integración social se entiende el sentido de pertenencia, por el cual el sujeto está integrado en unos grupos desempeñando un rol distinto al de cuidador.

Por otro lado, en el polo opuesto, haremos referencia a las cargas que supone el cuidado de un anciano, dentro de la familia. La carga o el peso fue considerado inicialmente como un tipo multideterminado de cansancio, incrementado por las nuevas responsabilidades adquiridas por los cuidadores (Cantor, 1983; Poulshock; Deimling, 1984; Robinson, 1983). George; Gwyther (1986), han definido la carga como los problemas fisiológicos, emocionales, sociales y económicos que pueden ser experimentados por los miembros de la familia que cuida de un anciano, como consecuencia de dichos cuidados.

Existen dos grandes aproximaciones teóricas, dentro del estudio de la carga: por un lado las perspectivas de estrés (Lawton; Kleban; Moss; Rovine; Glicksman, 1989; Poulschock; Dimling, 1984). Según este enfoque, la carga depende de la valoración subjetiva del cuidador sobre la experiencia de su nиevo trabajo. En segundo lugar, la perspectiva del contexto social (Miller; Mcfall; Montgomery, 1991), se basa en la existencia de otros costes personales e interpersonales que pueden existir independientemente de la valoración cognitiva que los cuidadores realizan de su tarea. Uno de estos coste será el económico.

En función de todo esto, sería interesante poder examinar las relaciones 
entre los tipos de apoyo y los tipos de carga, tanto uno a uno como la interrelación entre todos ellos. Hablaremos de ello en los siguientes apartados de este capítulo.

\section{El entorno familiar en la problemática del abandono}

En la medida en que la ciencia avanza, nuestra esperanza de vida tal y como señalábamos al principio de este capítulo, se hace cada vez más larga, llegando a nuevas situaciones de convivencia familiar y a un proceso de envejecimiento en el marco de la familia, con los consiguientes cambios y trastornos que de ello se derivan. Dichos cambios en el entorno los resume Martínez (1997) en dos:

1 Las relaciones sociales cambian a lo largo de la vida, volviéndose más íntimas y centrándose en el ambiente familiar, llegando el anciano en la última etapa evolutiva a relacionarse fundamentalmente con su grupo familiar.

2 El grupo de pertenencia que más se mantiene con la vejez está formado básicamente por la familia consanguínea y por los componentes del hogar familiar.

Debido a esta proximidad, la familia puede ser el grupo que acepta pero también rechaza, que acoge pero también repudia a sus componentes en función de una serie compleja de variables. De este modo, vivir solo o acompañado es un importante indicador de integración social y de calidad de vida. Así, la mayoría de los sujetos mayores de 65 años que viven en residencias lo hacen con su cónyuge (el $2 \%$ de parejas en residencias públicas y el $1 \%$ en residencias privadas). Los que viven en su domicilio se distribuyen del siguiente modo (según datos recogidos por Fernández-Ballesteros, 1997): el 20\% viven solos en su domicilio; un $40 \%$ viven con su pareja; un $15 \%$ además de vivir con su pareja, comparten domicilio con sus hijos; un 3\% viven con sus hijos y/o nietos y un 5\% lo hacen con otros familiares.

El número de hijos parece ser determinante a la hora de elegir la residencia como lugar para vivir: así, las personas que viven en residencias informan que han tenido como media un hijo, frente a los tres hijos de media de los que viven con su familia. 
Pero frente a esto, nos encontramos con otro grupo de ancianos que son abandonados por sus familiares sin ninguna explicación. Casi todos los veranos nos podemos encontrar con noticias en los periódicos sobre abandonos de personas mayores, bien en hospitales, en comisarías o hasta en lugares tan insólitos como en las gasolineras. El procedimiento más habitual es el abandono en un hospital o residencia, al que los familiares llevan al anciano con la excusa de una dolencia, dejándolos incluso sin documentación para que tarden más en localizarlas.

No obstante, sigue existiendo un numeroso grupo de familias que se hacen cargo de sus progenitores, que trataremos de examinar a continuación. En cuanto al sexo, la mayor parte de las personas mayores que necesitan cuidados son mujeres (esto es lógico, pues la esperanza de vida de la mujer es superior a la del hombre) y la mayoría de los familiares que proveen asistencia a estos ancianos son también mujeres (Lee, 1992; Verbrugge, 1989). Por otro lado, según Hing (1987) también es más probable que las mujeres sean institucionalizadas en mayor medida que los hombres, y que sean cuidadas por algún hijo en lugar del esposo.

En cuanto a los cuidadores, éstos son en mayor medida los cónyuges, seguidos en segundo lugar por los hijos cuando el anciano es viudo o por otros familiares (hermanos o nietos) y en cuarto lugar vecinos o amigos (Stoller, 1992). Dentro de esto, las mujeres cuidan más a menudo de sus maridos que viceversa (Tennstedt; McKinlay; Sullivan, 1989), las hijas tres veces más que los hijos, según datos de Dwyer; Coward (1991) y las hermanas más que los hermanos (Matthews; Rosner, 1988). Y en un 80\% de los casos, las personas ancianas solteras eran cuidadas por otras mujeres mayores (Soldo; Myllyluoma, 1983).

Dwyer, Folts; Rosenberg (1994), piensan que las diferencias encontradas son debidas al hecho de que los hombres y las mujeres definen el contexto del cuidador de forma diferente. Las tareas normalmente asociadas con los cuidados son aquellas que también tradicionalmente, han sido consideradas como trabajos de mujeres.

En cuanto a la edad, es previsible que hacia el año 2065 el número de personas mayores de 65 años se duplique, y los mayores de 85 de años sean 
una sexta parte de la población. Además, en el momento actual, los estudios (Rivlin; Wiener; Hanley; Spence, 1988) revelan que los mayores de 85 años tienen muchas más posibilidades de ser institucionalizados que los ancianos más jóvenes. Himes (1992), calcula que en el año 2000 habrá un $20 \%$ de mujeres mayores de 85 años y solteras sin hijos, que constituirán un grupo de alto riesgo y necesitarán de servicios asistenciales. Así, la ausencia de los recursos más comunes de apoyo (hijos o maridos) implicará que prácticamente la única salida que les reste sea la institucionalización. Del mismo modo, los cuidadores de estas personas mayores de 85 años, serán ancianos, pudiendo darse el caso de hijos mayores de 60 años cuidando de sus padres.

\section{Resultados del estudio del riesgo de abandono}

De la investigación realizada por Clemente; Vidal; Reguera (1997), podemos extraer las siguientes conclusiones, sobre cuáles son las variables más implicadas en el riesgo de abandono de los ancianos. Veamos a continuación los aspectos más reseñables.

En primer lugar, no es posible llegar a considerar que la vida en una residencia sea una forma de "abandono" o "semiabandono", ya que la gran mayoría de los ancianos encuestados habían elegido por propia voluntad esa opción de vida. Eso sí, no sabemos qué es lo que le lleva a elegir esa situación frente al resto de posibilidades. Quizá la percepción de apoyo social de su entorno le fuercen a optar por esta salida.

En cuanto a las relaciones sociales, podemos estar de acuerdo con los autores citados anteriormente, que enfatizaban la importancia de las redes sociales y del bienestar en los ancianos. Así, podríamos hipotetizar que quizá una consecuencia de la existencia de relaciones familiares más intensas es la que conlleva a que los ancianos se queden a vivir en las casas de sus familiares, produciéndose además esta elección de mutuo acuerdo en la mayoría de los casos.

Por su parte, los ancianos que viven en residencias son los que peores redes sociales tienen, entendiendo por éstas fundamentalmente las que se derivan de los círculos de mayor importancia emocional, como es la familia. Creemos de hecho que, en muchas ocasiones, el poseer una red social familiar de apoyo 
tan defectuosa, puede facilitarle al anciano el tomar la decisión personal de irse a vivir a una residencia. Nos parece que este es un dato importante, ya que cabría pensar que serían los ancianos abandonados los que peor relación y red social familiar tendrían; no es así, sino que dicho apoyo social es más deficitario en los ancianos que viven en residencias.

Todo esto nos lleva a pensar que quizá no sea tan importante el número de relaciones sociales ni la extensión de las redes sociales en sí, sino la calidad de dichas relaciones. Este hecho se encuentra reflejado, por ejemplo, en el nivel de preocupaciones que manifiestan los ancianos. Así, más de la mitad de las personas encuestadas que viven en su casa se encuentran preocupados por su familia. Los ancianos que viven en residencia comparten la preocupación por la familia en lo referente a la salud de éstos, siendo los abandonados los que mayor preocupación muestran por su propia salud, relegando la de la familia a un segundo o tercer plano.

Por otro lado, cuanto mayor es la vinculación con la familia, más son las preocupaciones que tienen, mientras que los ancianos desvinculados de sus familiares son los que muestran un menor nivel de preocupación.

En definitiva, creemos que todas las variables se encuentran muy relacionadas entre sí, y la conexión de todas ellas es la que nos conduce a la predicción del abandono. No obstante, señalaremos cuáles son las variables de mayor peso:

- el tiempo que pasa con la familia;

— la satisfacción o felicidad que se experimenta en la situación en la que se encuentra;

- la capacidad de decisión sobre la forma como se desea vivir.

— las preocupaciones por los demás (familia, amigos) y por uno mismo (salud, dinero y soledad);

— por último, en algunos casos, la práctica religiosa.

Todavía queda mucho por estudiar y saber en un tema incipiente, pero cada vez más puntero, como es el de las relaciones sociales entre la familia y los ancianos. Esta es una pequeña contribución, que a pesar de las posibles limitaciones y lagunas, consideramos de gran valor para seguir en esta línea de trabajo en futuras investigaciones. 


\section{Referencias bibliográficas}

1 AYUNTAMIENTO DE MADRID. La imagen de los ancianos entre los escolares. Madrid, 1993.

2 BROWN, G. W.; ANDREWS, B. Social Support and Depresion. In: APPLEY, M. H.; TRUMBULL, R. (Eds.). Dynamics of Stress. New York, Plenum Press, 1986.

3 CANTOR, M. H. Strain Among Caregivers: a study of experience in the United States. The Gerontologist, Washington, D.C., v.23, p.372-381, 1983.

4 CLEMENTE, M.; VIDAL, M. A.; REGUERA, N. Determinación de factores de riesgo en el abandono de ancianos. Madrid, Fundación Largo Caballero, 1997.

5 COMUNIDAD DE MADRID. La tercera edad en Madrid: aspectos cuantitativos. Madrid, 1989.

6 DIETZ, T. L. Patterns of Intergenerational Assistance within the Mexican American Family: is the family taking care of the older generation's needs? Journal of Family Issues, New York, N. Y., v.16, p.344-356, 1995.

7 DUNKEL-SCHETTER, C.; BENNETT, T. L. Differentiating the Cognitive and Behavior Aspects of Social Support. In: SARASON, B.R.; SARASON, I. G.; PIERCE, G. (Eds.). Social Support: an international view. New York, Wiley, 1990.

8 DWYER, J. W.; COWARD, R. T. A Multivariate Comparison of the Involvement of Adult Sons versus Daughters in the Care of Impaired Parents. Journal of Gerontology: Social Sciences, Washington, D.C., v.46, p. 259-S269, 1991.

9 DWYER, J. W.; FOLTS, W. E.; ROSENBERG, E.Caregiving in Social Context. Educational Gerontology, Washington, D. C., v.20, p.615-631, 1994.

10 EL PAÍS. Anuario El País 1998. Madrid, 1998.

11 FERNÁNDEZ-BALLESTEROS, R. Calidad de vida en la vejez: condiciones diferenciales. Anuario de Psicología, n.73, p.89-104, 1997. 
12 GEORGE, K. L.; GWYTHER, L. P. Caregiver Well-being: a multidimensional examination of family caregivers of dement adults. The Gerontologist, Washington, D.C., v.26, p.253-269, 1986.

13 HIMES, C. L. Future Caregivers: projected family structures of older persons. Journal of Gerontology: Social Sciences, Washington, D.C., v.17, p.17S26, 1992.

14 HING, E. Use of Nursing Homes by the Elderly: preliminary data from the 1985 National Nursing Home Survey. Hyattsville, National Center for Health Statistics, 1987.

15 HOUSE, J.; UMBERSON, D.; LANDIS, K. Structures and Processes of Social Support. Annual Review of Sociology, Palo Alto, Cal., v.14, p.293-318, 1988.

16 LAWTON, M. P.; KLEBAN, M. H.; MOSS, M.; ROVINE, M.; GLICKSMAN, A. Measuring Caregiver Appraisal. Journal of Gerontology: Psychological Sciences, Washington, D.C., v.44, p.P61-P71, 1989.

17 LEE, G. R. Gender Differences in Family Caregiving: a fact in search of a theory. In: DWYER, J. W.; COWARD, R. T. (Eds.). Gender, Families, and Elder care. Newbury Park, Sage, 1992.

18 LÓPEZ JIMENEZ,A. Actividad física y envejecimiento. In: ROTHSCHILD, H. (Comp.) Factores de Riesgo en la Edad Avanzada. México, La Prensa Mexicana, Mexico, D. F., P. 205-216, 1993.

19 MARTÍNEZ, G. Las relaciones interpersonales en el marco de la familia: algunas reflexiones metodológicas. Anuario de Psicología, Barcelona, n.73, p.19-29, 1997.

20 MATTHEWS, S. H.; ROSNER, J. Shared Filial Responsability: the family a the family a the primary caregiver. Journal of Marriage and Family, Minneapolis. Ma., v.51, p. 185-195, 1988.

21 MATTHEWS, S. H.; WERKNER, J. E., DELANEY, P. J. Relative Contributions of Help by Employed and Nonemployed Sisters to their Elderly Parents. Journal of Gerontology: Social Sciences, Washington, D.C., v.44, p.36-S44, 1989. 
22 MILlER, B.; McFALL, S.; MONTGOMERY, A. The Impact of Elder Health, Caregiver Involvement, and Global Stress on Two Dimensions of Caregiver Burden. Journal of Gerontology: Social Sciences, Washington, D.C., v.46, p. S9-S19, 1991.

23 PEARLIN, L. I.; MULLAN, J. T.; SEMPLE, S. J.; SKAFF, M. M.Caregiving and the stress process: an overview of concepts and their measures. The Gerontologist, Washington, D.C., v.30, p.583-594, 1990.

24 PHILLIPS, L. R.; REMPUSHESKI, V. F.; MORRISON, E. Risk Factors for Abuse and Neglect among Older Adults. Canadian Journal on Aging, v.14, p.20-44, 1989.

25 POULSHOCK, S. W.; DEIMLING, G. T. Families Caring for Elders in Residence: issues in the measurement of burden. Journal of Gerontology, Washington, D.C., v.39, p.230-239, 1984.

26 RIVLIN, A. M.; WIENER, J. M.; HANLEY, R. J.; SPENCE, D. A. Caring for the Disabled Elderly: who will pay? Washington, Brookings Institution, 1988.

27 ROBINSON, B. Validation of a Caregivers Strain Index. Journal of Gerontology, Washington, D.C., v.38, p.344-348, 1983.

28 SOLDO, B.; MYLLYLUOMA, J. Caregivers Who Live with Dependent Elderly. The Gerontologist, v.23, p.605-611, 1983.

29 STOLLER, E. P. Gender Differences in the Experiences of Caregiving Spouses. In: DWYER, J. W.; COWARD, R. T (Eds.). Gender, Families, and Elder Care. Newbury Park, Sage, 1992.

30 STONE, R.; CAFFERATA, G. L.; SANGL, J. Caregivers of the Frail Elderly: a national profile. The Gerontologist, Washington, D.C., v.27, p.616626, 1987.

31 TENNSTEDT, S.; McKINLAY, J.; SULLIVAN, L. M. Informal Care for Frail Elders: the role of secondary caregivers. The Gerontologist, Washington, D.C., v.29, p.677-683, 1989.

32 THOMPSON, E. H.; FUTTERMAN, AM.; GALLAGHER-THOMPSON, D.; ROSE, J. M.; LOVETT, S. B. Social Support and Caregiving Burden in Family Caregivers of Frail Elders. Journal of Gerontology: Social Sciences, Washington, D.C., v.48, p. S245-S254, 1993. 
33 VERBRUGGE, L. M. Gender, Aging, and Health. In: MARKIDES, K.S. (Ed.), Aging and Health. Newbury Park, Sage, 1989.

34 WELLMAN, B. From Social Support to Social Network. In: SARASON,

I. G.; SARASON, B. R. (Eds.). Social Support: theory, research and applications. Dordrecht, Martinus Nijhoff Publishers, 1985.

35 WELLMAN, B.; WORTLEY, S. Different Strokes from Different Folks: community ties and social support. American Journal of Sociology, Chicago, III., v.96, p.558-588, 1990.

36 WILBER, K. H.; SPECHT, C. V. Prevalence and Predictors of Burnout among Adult Day Care Providers. The Journal of Applied Gerontology, Thousand Clerks, Cal., v.13, p.282-298, 1994. 